\title{
The tomato genome sequence provides insights into fleshy fruit evolution
}

\author{
The Tomato Genome Consortium*
}

Tomato (Solanum lycopersicum) is a major crop plant and a model system for fruit development. Solanum is one of the largest angiosperm genera ${ }^{1}$ and includes annual and perennial plants from diverse habitats. Here we present a high-quality genome sequence of domesticated tomato, a draft sequence of its closest wild relative, Solanum pimpinellifolium ${ }^{2}$, and compare them to each other and to the potato genome (Solanum tuberosum). The two tomato genomes show only $\mathbf{0 . 6 \%}$ nucleotide divergence and signs of recent admixture, but show more than $8 \%$ divergence from potato, with nine large and several smaller inversions. In contrast to Arabidopsis, but similar to soybean, tomato and potato small RNAs map predominantly to gene-rich chromosomal regions, including gene promoters. The Solanum lineage has experienced two consecutive genome triplications: one that is ancient and shared with rosids, and a more recent one. These triplications set the stage for the neofunctionalization of genes controlling fruit characteristics, such as colour and fleshiness.

The genome of the inbred tomato cultivar 'Heinz 1706' was sequenced and assembled using a combination of Sanger and 'next generation' technologies (Supplementary Information section 1). The predicted genome size is approximately 900 megabases $(\mathrm{Mb})$, consistent with previous estimates ${ }^{3}$, of which $760 \mathrm{Mb}$ were assembled in 91 scaffolds aligned to the 12 tomato chromosomes, with most gaps restricted to pericentromeric regions (Fig. 1A and Supplementary Fig. 1). Base accuracy is approximately one substitution error per 29.4 kilobases $(\mathrm{kb})$ and one indel error per $6.4 \mathrm{~kb}$. The scaffolds were linked with two bacterial artificial chromosome (BAC)-based physical maps and anchored/oriented using a high-density genetic map, introgression line mapping and BAC fluorescence in situ hybridization (FISH).

The genome of S. pimpinellifolium LA1589 was sequenced and assembled de novo using Illumina short reads, yielding a $739 \mathrm{Mb}$ draft genome (Supplementary Information section 3). Estimated divergence between the wild and domesticated genomes is $0.6 \%$ (5.4 million single nucleotide polymorphisms (SNPs) distributed along the chromosomes (Fig. 1A and Supplementary Fig. 1)).

Tomato chromosomes consist of pericentric heterochromatin and distal euchromatin, with repeats concentrated within and around centromeres, in chromomeres and at telomeres (Fig. 1A and Supplementary Fig. 1). Substantially higher densities of recombination, genes and transcripts are observed in euchromatin, whereas chloroplast insertions (Supplementary Information sections 1.22 and 1.23) and conserved microRNA (miRNA) genes (Supplementary Information section 2.9) are more evenly distributed throughout the genome. The genome is highly syntenic with those of other economically important Solanaceae (Fig. 1B). Compared to the genomes of Arabidopsis ${ }^{4}$ and Sorghum ${ }^{5}$, tomato has fewer high-copy, full-length long terminal repeat (LTR) retrotransposons with older average insertion ages (2.8 versus 0.8 million years (Myr) ago) and fewer high-frequency $k$-mers (Supplementary Information section 2.10). This supports previous findings that the tomato genome is unusual among angiosperms by being largely comprised of low-copy $\mathrm{DNA}^{6,7}$.
The pipeline used to annotate the tomato and potato ${ }^{8}$ genomes is described in Supplementary Information section 2. It predicted 34,727 and 35,004 protein-coding genes, respectively. Of these, 30,855 and 32,988 , respectively, are supported by RNA sequencing (RNA-Seq) data, and 31,741 and 32,056, respectively, show high similarity to Arabidopsis genes (Supplementary Information section 2.1). Chromosomal organization of genes, transcripts, repeats and small RNAs (sRNAs) is very similar in the two species (Supplementary Figs 2-4). The proteincoding genes of tomato, potato, Arabidopsis, rice and grape were clustered into 23,208 gene groups ( $\geq 2$ members), of which 8,615 are common to all five genomes, 1,727 are confined to eudicots (tomato, potato, grape and Arabidopsis), and 727 are confined to plants with fleshy fruits (tomato, potato and grape) (Supplementary Information section 5.1 and Supplementary Fig. 5). Relative expression of all tomato genes was determined by replicated strand-specific Illumina RNA-Seq of root, leaf, flower (two stages) and fruit (six stages) in addition to leaf and fruit (three stages) of S. pimpinellifolium (Supplementary Table 1).

sRNA sequencing data supported the prediction of 96 conserved miRNA genes in tomato and 120 in potato, a number consistent with other plant species (Fig. 1A, Supplementary Figs 1 and 3 and Supplementary Information section 2.9). Among the 34 miRNA families identified, 10 are highly conserved in plants and similarly represented in the two species, whereas other, less conserved families are more abundant in potato. Several miRNAs, predicted to target Toll interleukin receptor, nucleotide-binding site and leucine-rich repeat (TIRNBS-LRR) genes, seemed to be preferentially or exclusively expressed in potato (Supplementary Information section 2.9).

Comparative genomic studies are reported in Supplementary Information section 4 . Sequence alignment of $71 \mathrm{Mb}$ of euchromatic tomato genomic DNA to their potato ${ }^{8}$ counterparts revealed $8.7 \%$ nucleotide divergence (Supplementary Information section 4.1). Intergenic and repeat-rich heterochromatic sequences showed more than $30 \%$ nucleotide divergence, consistent with the high sequence diversity in these regions among potato genotypes ${ }^{8}$. Alignment of tomato-potato orthologous regions confirmed nine large inversions known from cytological or genetic studies and several smaller ones (Fig. 1C). The exact number of small inversions is difficult to determine due to the lack of orientation of most potato scaffolds.

A total of 18,320 clearly orthologous tomato-potato gene pairs were identified. Of these, $138(0.75 \%)$ had significantly higher than average non-synonymous $\left(K_{\mathrm{a}}\right)$ versus synonymous $\left(K_{\mathrm{s}}\right)$ nucleotide substitution rate ratios $(\omega)$, indicating diversifying selection, whereas 147 $(0.80 \%)$ had significantly lower than average $\omega$, indicating purifying selection (Supplementary Table 2). The proportions of high and low $\omega$ between sorghum and maize (Zea mays) are $0.70 \%$ and $1.19 \%$, respectively, after 11.9 Myr of divergence ${ }^{9}$, indicating that diversifying selection may have been stronger in tomato-potato. The highest densities of low- $\omega$ genes are found in collinear blocks with average $K_{\mathrm{s}}>1.5$, tracing to a genome triplication shared with grape (see below) (Fig. 1C, Supplementary Fig. 6 and Supplementary Table 3). These genes, which have been preserved in paleo-duplicated locations for more than $100 \mathrm{Myr}^{10,11}$, are more constrained than 'average' genes and are

*Lists of participants and their affiliations appear at the end of the paper. 

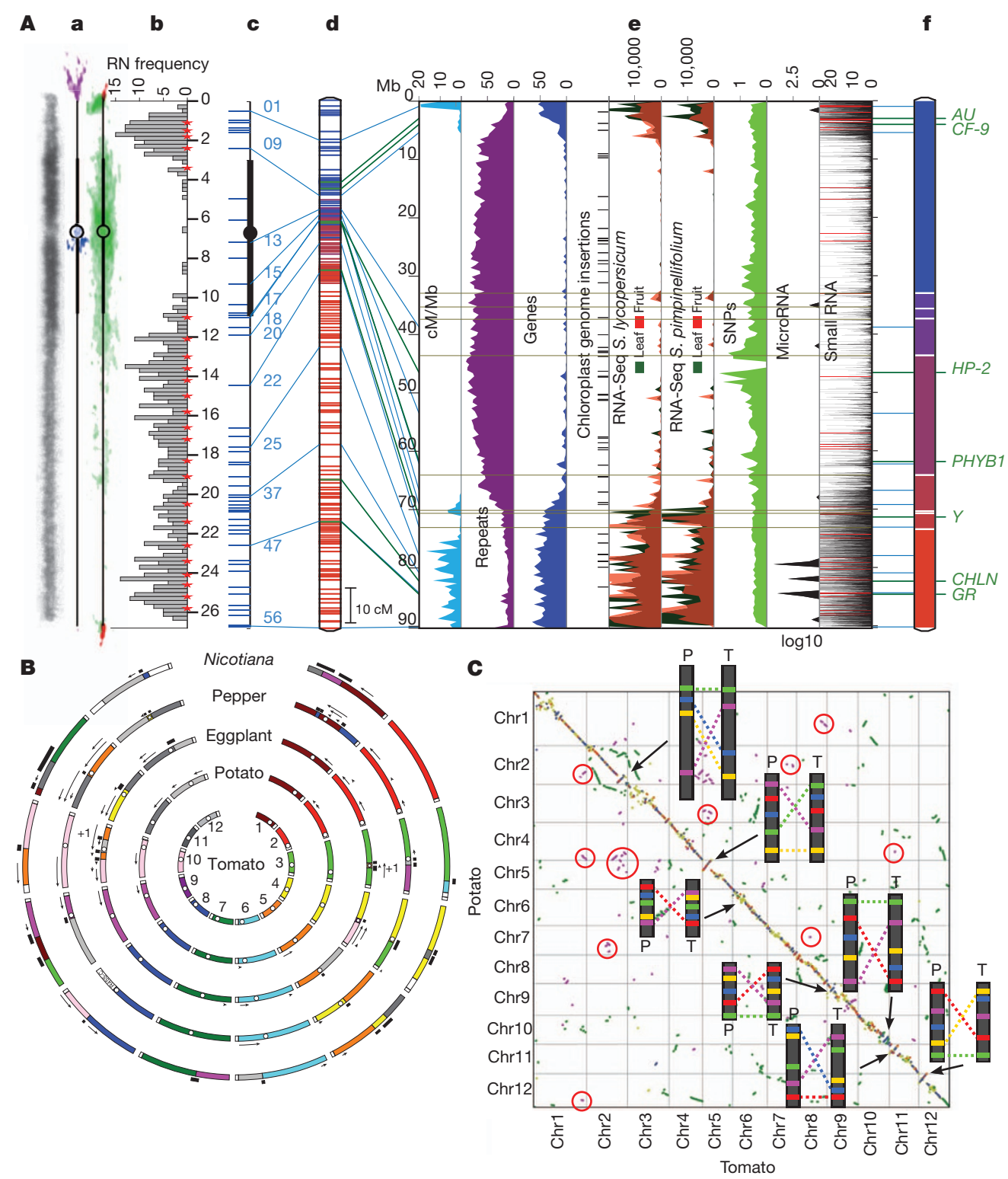

Figure $1 \mid$ Tomato genome topography and synteny. A, Multi-dimensional topography of tomato chromosome 1 (chromosomes 2-12 are shown in Supplementary Fig. 1). a, Left: contrast-reversed, 4',6-diamidino-2-

phenylindole (DAPI)-stained pachytene chromosome; centre and right: FISH signals for repeat sequences on diagrammatic pachytene chromosomes (purple, TGR1; blue, TGR4; red, telomere repeat; green, Cot 100 DNA (including most repeats)). $\mathbf{b}$, Frequency distribution of recombination nodules (RNs) representing crossovers on 249 chromosomes. Red stars mark $5 \mathrm{cM}$ intervals starting from the end of the short arm (top). Scale is in micrometres. c, FISHbased locations of selected BACs (horizontal blue lines on left). d, Kazusa F22000 linkage map. Blue lines to the left connect linkage map markers on the BAC-FISH map (c), and to the right to heat maps (e) and the DNA pseudomolecule (f). e, From left to right: linkage map distance $(\mathrm{cM} / \mathrm{Mb}$, turquoise), repeated sequences (\% nucleotides per $500 \mathrm{~kb}$, purple), genes (\% nucleotides per $500 \mathrm{~kb}$, blue), chloroplast insertions; RNA-Seq reads from leaves and breaker fruits of S. lycopersicum and S. pimpinellifolium (number of reads per $500 \mathrm{~kb}$, green and red, respectively), microRNA genes (transcripts per million per $500 \mathrm{~kb}$, black), small RNAs (thin horizontal black and red lines, sum of hits-normalized abundances). Horizontal grey lines represent gaps in the pseudomolecule (f). f, DNA pseudomolecule consisting of nine scaffolds. Unsequenced gaps (approximately $9.8 \mathrm{Mb}$, Supplementary Table 13) are

enriched for transcription factors and genes otherwise related to gene regulation (Supplementary Tables 3 and 4).

Sequence comparison of 31,760 Heinz 1706 genes with $>5 \times$ S. pimpinellifolium read coverage in over $90 \%$ of their coding regions indicated by white horizontal lines. Tomato genes identified by map-based cloning (Supplementary Table 14) are indicated on the right. For more details, see legend to Supplementary Fig. 1. B, Syntenic relationships in the Solanaceae. COSII-based comparative maps of potato, aubergine (eggplant), pepper and Nicotiana with respect to the tomato genome (Supplementary Information section 4.5 and Supplementary Fig. 14). Each tomato chromosome is assigned a different colour and orthologous chromosome segment(s) in other species are shown in the same colour. White dots indicate approximate centromere locations. Each black arrow indicates an inversion relative to tomato and ' +1 ' indicates a minimum of one inversion. Each black bar beside a chromosome indicates translocation breakpoints relative to tomato. Chromosome lengths are not to scale, but segments within chromosomes are. C, Tomato-potato syntenic relationships dot plot of tomato $(\mathrm{T})$ and potato $(\mathrm{P})$ genomic sequences based on collinear blocks (Supplementary Information section 4.1). Red and blue dots represent gene pairs with statistically significant high and low $\omega\left(K_{\mathrm{a}}\right)$ $K_{\mathrm{s}}$ ) in collinear blocks, which average $K_{\mathrm{s}} \leq 0.5$, respectively. Green and magenta dots represent genes in collinear blocks which average $0.5<K_{\mathrm{s}} \leq 1.5$ and $K_{\mathrm{s}}>1.5$, respectively. Yellow dots represent all other gene pairs. Blocks circled in red are examples of pan-eudicot triplication. Inserts represent schematic drawings of BAC-FISH patterns of cytologically demonstrated chromosome inversions (also in Supplementary Fig. 15).

revealed 7,378 identical genes and 11,753 with only synonymous changes. The remaining 12,629 genes had non-synonymous changes, including gains and losses of stop codons with potential consequences for gene function (Supplementary Tables 5-7). Several pericentric 
regions, predicted to contain genes, are absent or polymorphic in the broader S. pimpinellifolium germplasm (Supplementary Table 8 and Supplementary Fig. 7). Within cultivated germplasm, particularly among the small-fruited cherry tomatoes, several chromosomal segments are more closely related to $S$. pimpinellifolium than to Heinz 1706 (Supplementary Figs 8 and 9), supporting previous observations on recent admixture of these gene pools due to breeding ${ }^{12}$. Heinz 1706 itself has been reported to carry introgressions from S. pimpinellifolium ${ }^{13}$, traces of which are detectable on chromosomes 4, 9, 11 and 12 (Supplementary Table 9).

Comparison of the tomato and grape genomes supports the hypothesis that a whole-genome triplication affecting the rosid lineage occurred in a common eudicot ancestor ${ }^{11}$ (Fig. 2a). The distribution of $K_{\mathrm{s}}$ between corresponding gene pairs in duplicated blocks suggests that one polyploidization in the solanaceous lineage preceded the rosid-asterid (tomato-grape) divergence (Supplementary Fig. 10).

Comparison with the grape genome also reveals a more recent triplication in tomato and potato. Whereas few individual tomato/ potato genes remain triplicated (Supplementary Tables 10 and 11), $73 \%$ of tomato gene models are in blocks that are orthologous to one grape region, collectively covering $84 \%$ of the grape gene space. Among these grape genomic regions, $22.5 \%$ have one orthologous region in tomato, $39.9 \%$ have two, and $21.6 \%$ have three, indicating that a whole-genome triplication occurred in the Solanum lineage, followed by widespread gene loss. This triplication, also evident in potato (Supplementary Fig. 11), is estimated at 71 ( \pm 19.4$)$ Myr on the basis of the $K_{\mathrm{s}}$ of paralogous genes (Supplementary Fig. 10), and therefore predates the $\sim 7.3 \mathrm{Myr}$ tomato-potato divergence. On the basis of alignments to single grape genome segments, the tomato genome can be partitioned into three non-overlapping 'subgenomes' (Fig. 2b). The number of euasterid lineages that have experienced the recent triplication remains unclear and awaits complete euasterid I and II genome sequences. $K_{s}$ distributions show that euasterids I and II, and indeed the rosid-asterid lineages, all diverged from common ancestry at or near the pan-eudicot triplication (Fig. 2a), suggesting that this event may have contributed to the formation of major eudicot lineages in a short period of several million years ${ }^{14}$, partially explaining the explosive radiation of angiosperm plants on Earth ${ }^{15}$.

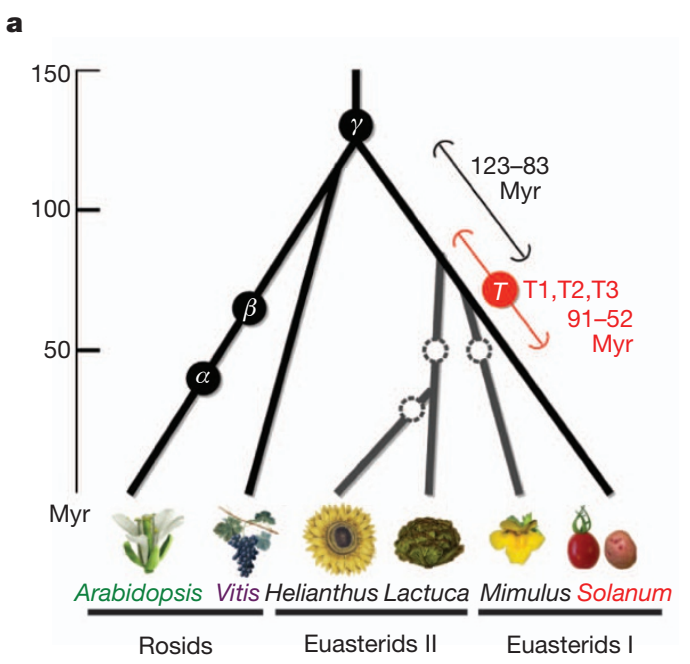

Figure 2 The Solanum whole genome triplication. a, Speciation and polyploidization in eudicot lineages. Confirmed whole-genome duplications and triplications are shown with annotated circles, including ' $T$ ' (this paper) and previously discovered events $\alpha, \beta, \gamma^{10,11,14}$. Dashed circles represent one or more suspected polyploidies reported in previous publications that need further support from genome assemblies ${ }^{27,28}$. Grey branches indicate unpublished genomes. Black and red error bars bracket indicate the likely timings of divergence of major asterid lineages and of ' $T$ ', respectively. The post-' $T$ ' subgenomes, designated T1, T2, and T3, are further detailed in
Fleshy fruits (Supplementary Fig. 12) are an important means of attracting vertebrate frugivores for seed dispersal ${ }^{16}$. Combined orthology and synteny analyses indicate that both genome triplications added new gene family members that mediate important fruit-specific functions (Fig. 3). These include transcription factors and enzymes necessary for ethylene biosynthesis (RIN, CNR, ACS) and perception $(E T R 3 / N R, E T R 4)^{17}$, red light photoreceptors influencing fruit quality (PHYB1/PHYB2) and ethylene- and light-regulated genes mediating lycopene biosynthesis (PSY1/PSY2). Several cytochrome P450 subfamilies associated with toxic alkaloid biosynthesis show contraction or complete loss in tomato and the extant genes show negligible expression in ripe fruits (Supplementary Information section 5.4).

Fruit texture has profound agronomic and sensory importance and is controlled in part by cell wall structure and composition ${ }^{18}$. More than 50 genes showing differential expression during fruit development and ripening encode proteins involved in modification of cell wall architecture (Fig. 4a and Supplementary Information section 5.7). For example, a family of xyloglucan endotransglucosylase/hydrolases (XTHs) has expanded both in the recent whole-genome triplication and through tandem duplication. One of the triplicated members, XTH10, shows differential loss between tomato and potato (Fig. 4a and Supplementary Table 12), suggesting genetically driven specialization in the remodelling of fruit cell walls.

Similar to soybean and potato and in contrast to Arabidopsis, tomato sRNAs map preferentially to euchromatin (Supplementary Fig. 2). sRNAs from tomato flowers and fruits ${ }^{19}$ map to 8,416 gene promoters. Differential expression of sRNAs during fruit development is apparent for 2,687 promoters, including those of cell-wall-related genes (Fig. 4b) and occurs preferentially at key developmental transitions (for example, flower to fruit, fruit growth to fruit ripening, Supplementary Information section 2.8).

The genome sequences of tomato, S. pimpinellifolium and potato provide a starting point for comparing gene family evolution and sub-functionalization in the Solanaceae. A striking example is the SELF PRUNING (SP) gene family, which includes the homologue of Arabidopsis FT, encoding the mobile flowering signal florigen ${ }^{20}$ and its antagonist SP, encoding the orthologue of TFL1. Nearly a century ago, a spontaneous mutation in $S P$ spawned the 'determinate' varieties

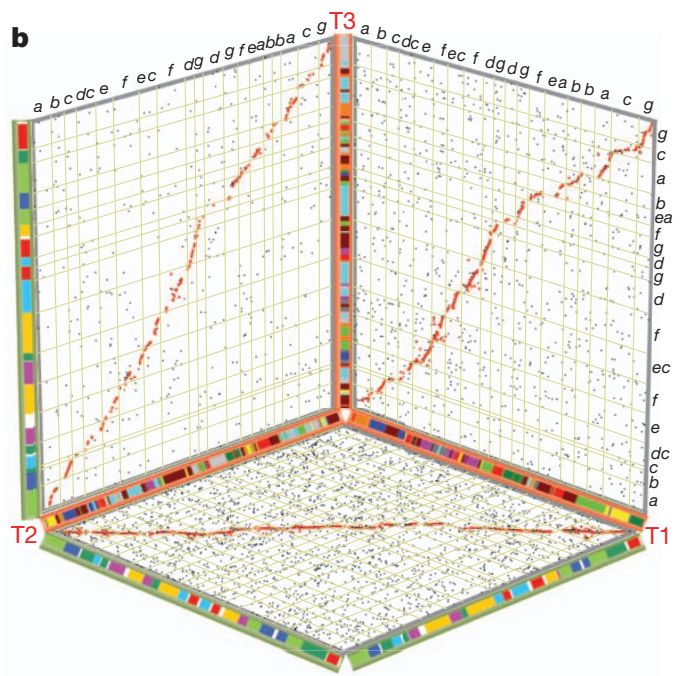

Supplementary Fig. 10. b, On the basis of alignments of multiple tomato genome segments to single grape genome segments, the tomato genome is partitioned into three non-overlapping 'subgenomes' (T1, T2, T3), each represented by one axis in the three-dimensional plot. The ancestral gene order of each subgenome is inferred according to orthologous grape regions, with tomato chromosomal affinities shown by red (inner) bars. Segments tracing to pan-eudicot triplication $(\gamma)$ are shown by green (outer) bars with colours representing the seven putative pre- $\gamma$ eudicot ancestral chromosomes ${ }^{10}$, also coded $a-g$. 


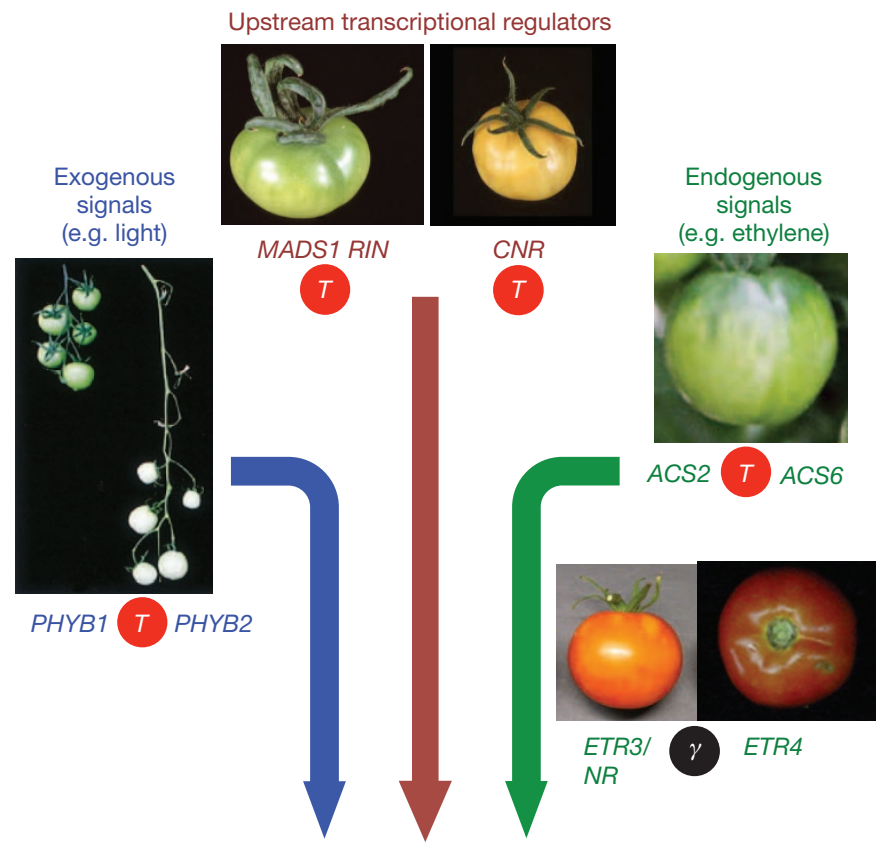

Downstream fruit development and ripening traits

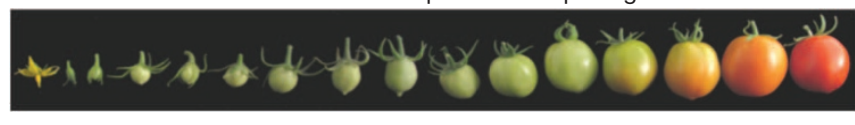

- Texture

- Aroma
Flavour

- Pigmentation

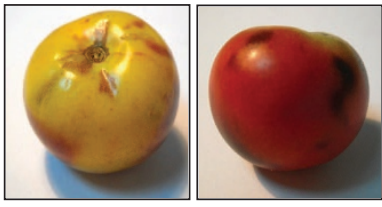

PSY1
- Pathogen susceptibility

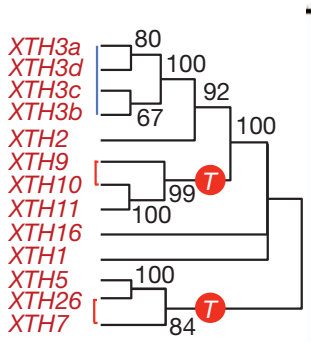

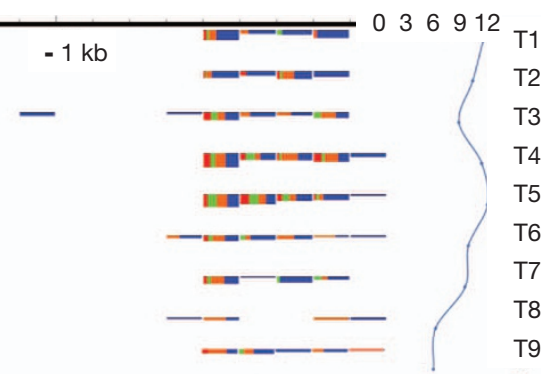

Figure $4 \mid$ The tomato genome allows systems approaches to fruit biology. a, Xyloglucan transglucosylase/hydrolases (XTHs) differentially expressed between mature green and ripe fruits (Supplementary Information section 5.7). These $X T H$ genes and many others are expressed in ripening fruits and are linked with the Solanum triplication, marked with a red circle on the phylogenetic tree. Red lines on the tree denote paralogues derived from the Solanum triplication, and blue lines are tandem duplications. b, Developmentally regulated accumulation of sRNAs mapping to the promoter region of a fruit-regulated cell wall gene (pectin acetylesterase, Solyc08g005800). Variation of abundance of sRNAs (left) and messenger RNA expression levels from the corresponding gene (right) over a tomato fruit developmental series (T1, bud; T2, flower; T3, fruit 1-3 mm; T4, fruit 5-7 mm; T5, fruit $11-13 \mathrm{~mm}$; 6 , fruit mature green; T7, breaker; T8, breaker +3 days; $\mathrm{T} 9$, breaker +7 days). The promoter regions are grouped in 100-nucleotide windows. For each window the size class distribution of sRNAs is shown (red, 21; green, 22; orange, 23; blue, 24). The height of the box corresponding to the first time point shows the cumulative sRNA abundance in log scale. The height of the following boxes is proportional to the log offset fold change ( offset $=20$ ) relative to the first time point. The expression profile of the mRNA is shown in $\log _{2}$ scale. The horizontal black line represents $1 \mathrm{~kb}$ of the promoter region. 0 to 12 represent arbitrary units of gene expression.

the 16th century, and the intensive breeding that followed. Charles Rick pioneered the use of trait introgression from wild tomato relatives to increase genetic diversity of cultivated tomatoes ${ }^{26}$. Introgression lines exist for seven wild tomato species, including S. pimpinellifolium, in the background of cultivated tomato. The genome sequences presented here and the availability of millions of SNPs will allow breeders to revisit this rich trait reservoir and identify domestication genes, providing biological knowledge and empowering biodiversity-based breeding.

\section{METHODS SUMMARY}

A total of 21 gigabases $(\mathrm{Gb})$ of Roche/454 Titanium shotgun and mate pair reads and $3.3 \mathrm{~Gb}$ of Sanger paired-end reads, including $\sim 200,000 \mathrm{BAC}$ and fosmid end sequence pairs, were generated from the 'Heinz 1706' inbred line (Supplementary Information sections 1.1-1.7), assembled using both Newbler and CABOG and integrated into a single assembly (Supplementary Information sections 1.17 and 1.18). The scaffolds were anchored using two BAC-based physical maps, one high density genetic map, overgo hybridization and genome-wide BAC FISH (Supplementary Information sections 1.8-1.16 and 1.19). Over 99.9\% of BAC/ fosmid end pairs mapped consistently on the assembly and over $98 \%$ of EST sequences could be aligned to the assembly (Supplementary Information section 1.20). Chloroplast genome insertions in the nuclear genome were validated using a mate pair method and the flanking regions were identified (Supplementary Information sections 1.22-1.24). Annotation was carried out using a pipeline based on EuGene that integrates de novo gene prediction, RNA-Seq alignment and rich function annotation (Supplementary Information section 2). To facilitate interspecies comparison, the potato genome was re-annotated using the same pipeline. LTR retrotransposons were detected de novo with the LTR-STRUC program and dated by the sequence divergence between left and right solo LTR (Supplementary Information section 2.10). The genome of S. pimpinellifolium was sequenced to $\times 40$ depth using Illumina paired end reads and assembled using ABySS (Supplementary Information section 3). The tomato and potato genomes were aligned using LASTZ (Supplementary Information section 4.1). Identification of triplicated regions was done using BLASTP, in-house-generated scripts and three-way comparisons between tomato, potato and S. pimpinellifolium using MCSCAN (Supplementary Information sections 4.2-4.4). Specific gene families/ tomato genetic diversity: the domestication of S. pimpinellifolium in the Americas, the export of a small number of genotypes to Europe in 
groups (genes for ascorbate, carotenoid and jasmonate biosynthesis, cytochrome P450s, genes controlling cell wall architecture, hormonal and transcriptional regulators, resistance genes) were subjected to expert curation/analysis (Supplementary Information section 5). PHYML and MEGA were used to reconstruct phylogenetic trees and MCSCAN was used to infer gene collinearity (Supplementary Information section 5.2).

\section{Received 17 August 2011; accepted 3 April 2012.}

1. Frodin, D. G. History and concepts of big plant genera. Taxon 53, 753-776 (2004)

2. Peralta, I. E., Spooner, D. M. \& Knapp, S. Taxonomy of tomatoes: a revision of wild tomatoes (Solanum section Lycopersicon) and their outgroup relatives in sections Juglandifolia and Lycopersicoides. Syst. Bot. Monogr. 84, 1-186 (2008)

3. Michaelson, M. J., Price, H. J., Ellison, J. R. \& Johnston, J. S. Comparison of plant DNA contents determined by Feulgen microspectrophotometry and laser flow cytometry. Am. J. Bot. 78, 183-188 (1991)

4. The Arabidopsis Genome Initiative. Analysis of the genome sequence of the flowering plant Arabidopsis thaliana. Nature 408, 796-815 (2000).

5. Paterson, A. H. etal. The Sorghum bicolor genome and the diversification of grasses. Nature 457, 551-556 (2009).

6. Zamir, D. \& Tanksley, S. D. Tomato genome is comprised largely of fast-evolving, low copy-number sequences. Mol. Gen. Genet. 213, 254-261 (1988).

7. Peterson, D. G., Pearson, W. R. \& Stack, S. M. Characterization of the tomato (Lycopersicon esculentum) genome using in vitro and in situ DNA reassociation. Genome 41, 346-356 (1998).

8. Xu, X. et al. Genome sequence and analysis of the tuber crop potato. Nature $\mathbf{4 7 5}$, 189-195 (2011).

9. Swigoňová, Z.etal. Close split of sorghum and maize genome progenitors. Genome Res. 14, 1916-1923 (2004).

10. Jaillon, 0 et al The grapevine genome sequence suggests ancestral hexaploidization in major angiosperm phyla. Nature 449, 463-467 (2007).

11. Tang, H. et al. Synteny and collinearity in plant genomes. Science 320, 486-488 (2008).

12. Ranc, N., Munos, S., Santoni, S. \& Causse, M. A clarified position for Solanum lycopersicum var. cerasiforme in the evolutionary history of tomatoes (solanaceae). BMC Plant Biol. 8, 130 (2008).

13. Ozminkowski, R. Pedigree of variety Heinz 1706. Rep. Tomato Genet. Coop. 54, 26 (2004).

14. Moore, M. J., Soltis, P. S., Bell, C. D., Burleigh, J. G. \& Soltis, D. E. Phylogenetic analysis of 83 plastid genes further resolves the early diversification of eudicots. Proc. Natl Acad. Sci. USA 107, 4623-4628 (2010).

15. Stockey, R. A., Graham, S. W. \& Crane, P. R. Introduction to the Darwin special issue: the abominable mystery. Am. J. Bot. 96, 3-4 (2009).

16. Howe, H. F. \& Smallwood, J. Ecology of seed dispersal. Annu. Rev. Ecol. Syst. 13 201-228 (1982).

17. Klee, H. J. \& Giovannoni, J. J. Genetics and control of tomato fruit ripening and quality attributes. Annu. Rev. Genet. 45, 41-59 (2011).

18. Vicente, A. R., Saladie, M., Rose, J. K. C. \& Labavitch, J. M. The linkage between cell wall metabolism and fruit softening: looking to the future. J. Sci. Food Agric. $\mathbf{8 7}$ 1435-1448 (2007)

19. Mohorianu, I. et al. Profiling of short RNAs during fleshy fruit development reveals stage-specific sRNAome expression patterns. Plant J. 67, 232-246 (2011).

20. Corbesier, L. et al. FT protein movement contributes to long-distance signaling in floral induction of Arabidopsis. Science 316, 1030-1033 (2007).

21. Rick, C. M. The tomato. Sci. Am. 239, 76-87 (1978).

22. Navarro, C. et al. Control of flowering and storage organ formation in potato by FLOWERING LOCUS T. Nature 478, 119-122 (2011).

23. Lifschitz, E. et al. The tomato FT ortholog triggers systemic signals that regulate growth and flowering and substitute for diverse environmental stimuli. Proc. Nat Acad. Sci. USA 103, 6398-6403 (2006).

24. Krieger, U., Lippman, Z. B. \& Zamir, D. The flowering gene SINGLE FLOWER TRUSS drives heterosis for yield in tomato. Nature Genet. 42, 459-463 (2010).

25. Pnueli, L. et al. The SELF-PRUNING gene of tomato regulates vegetative to reproductive switching of sympodial meristems and is the ortholog of CEN and TFL1. Development 125, 1979-1989 (1998).

26. Rick, C. M. Hybridization between Lycopersicon esculentum and Solanum pennellii: phylogenetic and cytogenetic significance. Proc. Natl Acad. Sci. USA 46, 78-82 (1960)

27. Barker, M. S. et al. Multiple paleopolyploidizations during the evolution of the Compositae reveal parallel patterns of duplicate gene retention after millions of years. Mol. Biol. Evol. 25, 2445-2455 (2008).

28. Aagaard, J. E., Willis, J. H. \& Phillips, P. C. Relaxed selection among duplicate floral regulatory genes in Lamiales. J. Mol. Evol. 63, 493-503 (2006).

Supplementary Information is linked to the online version of the paper at www.nature.com/nature.

Acknowledgements This work was supported by: Argentina: INTA and CONICET. Belgium: Flemish Institute for Biotechnology and Ghent University. China: The State Key Laboratory of Plant Genomics, Institute of Genetics and Developmental Biology, Chinese Academy of Sciences: Ministry of Science and Technology (2006AA10A116, 2004CB720405, 2006CB101907, 2007DFB30080) Ministry of Agriculture ('948' Program: 2007-Z5); National Natural Science Foundation (36171319); Postdoctoral Science Foundation (20070420446). European Union: FP6 Integrated ProjectEU-SOL PL 016214 . France: Institute National de la Recherche Agronomique and Agence Nationale de la Recherche. Germany: the Max Planck Society. India: Department of
Biotechnology, Government of India; Indian Council of Agricultural Research. Italy: Ministry of Research (FIRB-SOL, FIRB-Parallelomics, ItaLyco and GenoPOM projects); Ministry of Agriculture (Agronanotech and Biomassval projects); FILAS foundation; ENEA: CNR-ENEA project L. 191/2009. Japan: Kazusa DNA Research Institute Foundation and National Institute of Vegetable and Tea Science. Korea: KRIBB Basic Research Fund and Crop Functional Genomics Research Center (CFGC), MEST. Netherlands: Centre for BioSystems Genomics, Netherlands Organization for Scientific Research. Spain: Fundación Genoma España; Cajamar; FEPEX; Fundación Séneca; ICIA; IFAPA; Fundación Manrique de Lara; Instituto Nacional de Bioinformatica. UK: BBSRC grant BB/C509731/1: DEFRA; SEERAD. USA: NSF (DBI-0116076; DBI-0421634; DBI-0606595; IOS-0923312; DBI-0820612; DBI-0605659; DEB-0316614; DBI 0849896 and MCB 1021718); USDA (2007-02773 and 2007-35300-19739); USDA-ARS. We acknowledge the Potato Genome Sequencing Consortium for sharing data before publication; potato RNA-Seq data was provided by C. R. Buell from the NSF-funded Potato Genome Sequence and Annotation project; tomato RNA-Seq data by the USDA-funded SolCAP project, N. Sinha and J. Maloof; the Amplicon Express team for BAC pooling services; construction of the Whole Genome Profiling (WGP) physical map was supported by EnzaZaden, RijkZwaan, Vilmorin \& Cie and Takii \& Co. Keygene N.V. owns patents and patent applications covering its AFLP and Whole Genome Profiling technologies; AFLP and Keygene are registered trademarks of Keygene N.V. The following individuals are also acknowledged for their contribution to the work described: J. Park, B. Wang, C. Niu, D. Liu, F. Cojutti,

S. Pescarolo, A. Zambon, G. Xiao, J. Chen, J. Shi, L. Zhang, L. Zeng, M. Caccamo, D. Bolser, D. Martin, M. Gonzalez, P. A. Bedinger, P. A. Covey, P. Pachori, R. R. Pousada, S. Hakim S. Sims, V. Cahais, W. Long, X. Zhou, Y. Lu, W. Haso, C. Lai, S. Lepp, C. Peluso, H. Teramu H. De Jong. R. Lizarralde, E. R. May and Z. Li. M. Zabeau is thanked for his support and encouragement and S. van den Brink for her secretarial support. We dedicate this work to the late $\mathrm{C}$. Rick who pioneered tomato genetics, collection of wild germplasm and the distribution of seed and knowledge.

Author Contributions For full details of author contributions, please see the Supplementary Information.

Author Information The genomic data generated by the whole project are available in GenBank as accession number AEKE00000000, and the individual chromosome sequences as numbers CM001064-CM001075. The RNA-Seq data are available in the Sequence Read Archive under accession number SRA049915, GSE33507, SRA050797 and SRA048144. Further information on data access can be found in Supplementary Information section 2.2. Reprints and permissions information is available at www.nature.com/reprints. The authors declare no competing financia interests. This paper is distributed under the terms of the Creative Commons Attribution-Non-Commercial-Share Alike licence, and is freely available to all readers at www.nature.com/nature. Readers are welcome to comment on the online version of this article at www.nature.com/nature. Correspondence and requests for materials should be addressed to D.Z. (zamir@agri.huji.ac.il) or G.G. (giovanni.giuliano@enea.it)

\section{The Tomato Genome Consortium}

Kazusa DNA Research Institute Shusei Sato (Principal Investigator) ${ }^{1}$, Satoshi Tabata (Principal Investigator) ${ }^{1}$, Hideki Hirakawa ${ }^{1}$, Erika Asamizu ${ }^{1}$, Kenta Shirasawa $^{1}$, Sachiko Isobe ${ }^{1}$, Takakazu Kaneko ${ }^{1}$, Yasukazu Nakamura ${ }^{1}$, Daisuke Shibata ${ }^{1}$, Koh Aoki ${ }^{1}$; 454 Life Sciences, a Roche company Michael Egholm² ${ }^{2}$ James Knight ${ }^{2}$; Amplicon Express Inc. Robert Bogden ${ }^{3}$; Beijing Academy of Agriculture and Forestry Sciences Changbao Li $i^{4,5}$; BGI-Shenzhen Yang Shuang ${ }^{6}$, Xun $\mathrm{Xu}^{6}$, Shengkai Pan ${ }^{6}$

Shifeng Cheng ${ }^{6}$, Xin Liu' ${ }^{6}$, Yuanyuan Ren ${ }^{6}$, Jun Wang; ; BMR-Genomics SrL Alessandro Albiero ${ }^{7}$, Francesca Dal Pero 7 , Sara Todesco 7 ; Boyce Thompson Institute for Plant Research Joyce Van Eck ${ }^{8}$, Robert M. Buels ${ }^{8}$, Aureliano Bombarely ${ }^{8}$, Joseph R. Gosselin ${ }^{8}$, Minyun Huang 8 , Jonathan A. Leto ${ }^{8}$, Naama Menda ${ }^{8}$, Susan Strickler ${ }^{8}$, Linyong Mao ${ }^{8}$, Shan Gao ${ }^{8}$, Isaak Y. Tecle ${ }^{8}$, Thomas York ${ }^{8}$, Yi Zheng ${ }^{8}$, Julia T. Vrebalov $^{8}$, JeMin Lee ${ }^{8}$, Silin Zhong ${ }^{8}$, Lukas A. Mueller (Principal Investigator) ${ }^{8}$; Centre for BioSystems Genomics Willem J. Stiekema ${ }^{9}$; Centro Nacional de Análisis Genómico (CNAG) Paolo Ribeca ${ }^{10}$, Tyler Alioto ${ }^{10}$; China Agricultural University Wencai Yang ${ }^{11}$; Chinese Academy of Agricultural Sciences Sanwen Huang (Principal Investigator ${ }^{12}$, Yongchen Du (Principal Investigator) ${ }^{12}$, Zhonghua Zhang ${ }^{12}$ Jianchang Gao ${ }^{12}$, Yanmei Guo ${ }^{12}$, Xiaoxuan Wang ${ }^{12}$, Ying $\mathrm{Li}^{12}$, Jun $\mathrm{He}^{12}$; Chinese Academy of Sciences Chuanyou Li (Principal Investigator) ${ }^{13}$, Zhukuan Cheng (Principal Investigator) ${ }^{13}$, Jianru Zuo (Principal Investigator) ${ }^{13}$, Jianfeng Ren ${ }^{13}$, Jiuhai Zhao ${ }^{13}$, Liuhua Yan ${ }^{13}$, Hongling Jiang ${ }^{13}$, Bao Wang ${ }^{13}$, Hongshuang Li ${ }^{13}$, Zhenjun Li ${ }^{13}$, Fuyou $\mathrm{Fu}^{13}$, Bingtang Chen ${ }^{13}$, Bin Han (Principal Investigator) ${ }^{5}$, Qi Feng ${ }^{5}$, Danlin Fan ${ }^{5}$, Ying Wang (Principal Investigator) ${ }^{14}$, Hongqing Ling (Principal Investigator) ${ }^{15}$, Yongbiao Xue (Principal Investigator ${ }^{16}$; Cold Spring Harbor Laboratory and United States Department of Agriculture - Agricultural Research Service Doreen Ware (Principal Investigator) ${ }^{17}$, W. Richard McCombie (Principal Investigator) ${ }^{17}$, Zachary B. Lippman (Principal Investigator) $^{17}$, Jer-Ming Chia ${ }^{17}$, Ke Jiang ${ }^{17}$, Shiran Pasternak ${ }^{17}$, Laura Gelley ${ }^{17}$, Melissa Kramer ${ }^{17}$; Colorado State University Lorinda K. Anderson ${ }^{18}$, Song-Bin Chang ${ }^{19}$, Suzanne M. Royer ${ }^{18}$, Lindsay A. Shearer ${ }^{18}$, Stephen M. Stack (Principal Investigator) $^{18}$; Cornell University Jocelyn K. C. Rose ${ }^{20}$, Yimin $\mathrm{Xu}^{20}$, Nancy Eannetta ${ }^{20}$, Antonio J. Matas ${ }^{20}$, Ryan McQuinn ${ }^{20}$, Steven D. Tanksley (Principal Investigator ${ }^{20}$; Genome Bioinformatics Laboratory GRIB-IMIM/UPF/CRG Francisco Camara $^{21}$, Roderic Guigó ${ }^{21}$; Ghent University-VIB Stephane Rombauts ${ }^{22}$, Jeffrey Fawcett ${ }^{22}$, Yves Van de Peer (Principal Investigator) ${ }^{22}$; Hebrew University of Jerusalem Dani Zamir ${ }^{23}$; Heilongiliang Academy of Agricultural Sciences Chunbo Liang $^{24}$; Helmholtz Center for Health and Environment Manuel Spannagl ${ }^{25}$, Heidrun Gundlach $^{25}$, Remy Bruggmann ${ }^{25}$, Klaus Mayer (Principal Investigator) ${ }^{25}$; Henan 
Agricultural University Zhiqi Jia ${ }^{26}$; Huazhong Agricultural University Junhong Zhang ${ }^{27}$, Zhibiao Ye ${ }^{27}$; Imperial College London Gerard J. Bishop (Principal Investigator) ${ }^{28}$, Sarah Butcher (Principal Investigator) ${ }^{28}$, Rosa Lopez-Cobollo ${ }^{28}$, Daniel Buchan ${ }^{28}$, Ioannis Filippis ${ }^{28}$, James Abbott ${ }^{28}$; Indian Agricultural Research Institute Rekha Dixit ${ }^{29}$, Manju Singh ${ }^{29}$, Archana Singh ${ }^{29}$, Jitendra Kumar Pal ${ }^{29}$, Awadhesh Pandit ${ }^{29}$, Pradeep Kumar Singh ${ }^{29}$, Ajay Kumar Mahato ${ }^{29}$, Vivek Dogra ${ }^{29}$ Kishor Gaikwad ${ }^{29}$, Tilak Raj Sharma ${ }^{29}$, Trilochan Mohapatra $^{29}$, Nagendra Kumar Singh (Principal Investigator) ${ }^{29}$; INRA Avignon Mathilde Causse ${ }^{30}$; INRA Bordeaux Christophe Rothan ${ }^{31}$; INRA Toulouse Thomas Schiex (Principal Investigator) ${ }^{32}$, Céline Noirot ${ }^{32}$, Arnaud Bellec ${ }^{33}$, Christophe Klopp ${ }^{34}$, Corinne Delalande ${ }^{35}$, Hélène Berges $^{33}$, Jérôme Mariette ${ }^{34}$, Pierre Frasse ${ }^{35}$, Sonia Vautrin ${ }^{33}$; Institut National Polytechnique de Toulouse Mohamed Zouine ${ }^{35}$, Alain Latché ${ }^{35}$, Christine Rousseau $^{35}$, Farid Regad ${ }^{35}$, Jean-Claude Pech ${ }^{35}$, Murielle Philippot ${ }^{35}$, Mondher Bouzayen (Principal Investigator) ${ }^{35}$; Instituto de Biología Molecular y Celular de Plantas (CSIC-UPV) Pierre Pericard ${ }^{36}$, Sonia Osorio ${ }^{36}$, Asunción Fernandez del Carmen $^{36}$, Antonio Monforte ${ }^{36}$, Antonio Granell (Principal Investigator) ${ }^{36}$; Instituto de Hortofruticultura Subtropical y Mediterránea (IHSM-UMA-CSIC) Rafael Fernandez-Muñoz ${ }^{37}$; Instituto Nacional de Tecnología Agropecuaría (IB-INTA) and Consejo Nacionalde Investigaciones Científicas y Técnicas (CONICET) Mariana Conte $^{38}$, Gabriel Lichtenstein ${ }^{38}$, Fernando Carrari (Principal Investigator) ${ }^{38}$; Italian National Res Council, Institute for Biomedical Technologies Gianluca De Bellis (Principal Investigator) $^{39}$, Fabio Fuligni ${ }^{39}$, Clelia Peano ${ }^{39}$; Italian National Research Council, Institute of Plant Genetics, Research Division Portici Silvana Grandillo ${ }^{40}$, Pasquale Termolino ${ }^{40}$; Italian National Agency for New technologies, Energy and Sustainable Development Marco Pietrella $a^{41,42}$, Elio Fantini ${ }^{41}$, Giulia Falcone ${ }^{41}$, Alessia Fiore ${ }^{41}$, Giovanni Giuliano (Principal Investigator) ${ }^{41}$, Loredana Lopez ${ }^{43}$, Paolo Facella ${ }^{43}$, Gaetano Perrotta ${ }^{43}$, Loretta Daddiego ${ }^{43}$; James Hutton Institute Glenn Bryan (Principal Investigator) ${ }^{44}$; Joint IRB-BSC program on Computational Biology Modesto Orozco ${ }^{45,46}$, Xavier Pastor ${ }^{45}$, David Torrents ${ }^{45,47}$; Keygene N.V. Marco G. M. van Schriek ${ }^{48}$, Richard M.C. Feron ${ }^{48}$, Jan van Oeveren ${ }^{48}$, Peter de Heer $^{48}$, Lorena daPonte ${ }^{48}$, Saskia Jacobs-Oomen ${ }^{48}$, Mike Cariaso $0^{48}$, Marcel Prins ${ }^{48}$ Michiel J. T. van Eijk (Principal Investigator) ${ }^{48}$, Antoine Janssen ${ }^{48}$,'Mark J. J. van Haaren ${ }^{48}$; Korea Research Institute of Bioscience and Biotechnology Sung-Hwan Jo ${ }^{49}$ Jungeun $\mathrm{Kim}^{49}$, Suk-Yoon Kwon ${ }^{49}$, Sangmi Kim ${ }^{49}$, Dal-Hoe Koo ${ }^{49}$, Sanghyeob Lee $^{49}$;, Cheol-Goo Hur ${ }^{49}$; Life Technologies Christopher Clouser ${ }^{50}$, Alain Rico ${ }^{51}$; Max Planck Institute for Plant Breeding Research Asis Hallab ${ }^{52}$, Christiane Gebhardt ${ }^{52}$ Kathrin Klee ${ }^{52}$, Anika Jöcker ${ }^{52}$, Jens Warfsmann ${ }^{52}$, Ulrike Göbel ${ }^{52}$; Meiji University Shingo Kawamura ${ }^{53}$, Kentaro Yano ${ }^{53}$; Montana State University Jamie D. Sherman ${ }^{54}$; NARO Institute of Vegetable and Tea Science Hiroyuki Fukuoka (Principal

Investigator) ${ }^{55}$, Satomi Negoro ${ }^{55}$; National Institute of Plant Genome Research Sarita Bhutty $^{56}$, Parul Chowdhury ${ }^{56}$, Debasis Chattopadhyay (Principal Investigator) ${ }^{56}$, Plant Research International Erwin Datema ${ }^{48,57}$, Sandra Smit ${ }^{57}$, Elio G. W. M.

Schijlen $^{57}$, Jose van de Belt ${ }^{57}$, Jan C. van Haarst ${ }^{57}$, Sander A. Peters ${ }^{57}$, Marjo J. van Staveren $^{57}$, Marleen H. C. Henkens ${ }^{57}$, Paul J. W. Mooyman ${ }^{57}$, Thamara Hesselink ${ }^{57}$, Roeland C. H. J. van Ham (Principal Investigator) ${ }^{48,53}$; Qingdao Agricultural University Guoyong Jiang ${ }^{58}$; Roche Applied Science Marcus Droege ${ }^{59}$; Seoul National University Doil Choi (Principal Investigator) ${ }^{60}$, Byung-Cheol Kang ${ }^{60}$, Byung Dong $\mathrm{Kim}^{60}{ }^{6}$ Minkyu Park ${ }^{60}$, Seungill Kim ${ }^{60}$, Seon-In Yeom ${ }^{60}$, Yong-Hwan Lee ${ }^{61}$, Yang-Do Sichuan University Yongsheng Liu ${ }^{65}$, Shengxiong Huang ${ }^{65}$; Sistemas Genomicos
Victoria Fernandez-Pedrosa ${ }^{66}$, Carmen Collado ${ }^{66}$, Sheila Zuñiga ${ }^{66}$; South China Agricultural University Guoping Wang ${ }^{67}$; Syngenta Biotechnology Rebecca Cade ${ }^{68}$, Robert A. Dietrich ${ }^{68}$; The Genome Analysis Centre Jane Rogers (Principal Investigator $)^{69}$; The Natural History Museum Sandra Knapp ${ }^{70}$; United States Department of Agriculture-Agricultural Research Service, RobertW. Holley Center and Boyce Thompson Institute for Plant Research Zhangjun Fei (Principal

Investigator $)^{8,71}$, Ruth A. White ${ }^{8,71}$, Theodore W. Thannhauser ${ }^{8,71}$, James J. Giovannoni (Principal Investigator) ${ }^{8,20,71}$; Universidad de Malaga-Consejo Superior de Investigaciones Cientificas Miguel Angel Botella ${ }^{72}$, Louise Gilbert ${ }^{72}$; Universitat Pompeu Fabra Ramon Gonzalez ${ }^{73}$; University of Arizona Jose Luis Goicoechea ${ }^{74}$, Yeisoo $\mathrm{Yu}^{74}$, David Kudrna ${ }^{74}$, Kristi Collura ${ }^{74}$, Marina Wissotski ${ }^{74}$, Rod Wing (Principal Investigator) ${ }^{74}$; University of Bonn Heiko Schoof (Principal Investigator) ${ }^{75}$; University of Delaware Blake C. Meyers (Principal Investigator) ${ }^{76}$, Aishwarya Bala Gurazada $^{76}$, Pamela J. Green ${ }^{76}$; University of Delhi South Campus Saloni Mathur ${ }^{77}$ Shailendra Vyas ${ }^{77}$, Amolkumar U. Solanke ${ }^{77}$, Rahul Kumar ${ }^{77}$, Vikrant Gupta ${ }^{77}$, Arun K. Sharma 77 , Paramjit Khurana ${ }^{77}$, Jitendra P. Khurana (Principal Investigator) ${ }^{77}$, Akhilesh K. Tyagi (Principal Investigator) ${ }^{77}$; University of East Anglia, School of Biological Sciences Tamas Dalmay (Principal Investigator) ${ }^{78}$; University of East Anglia, School of Computing Sciences Irina Mohorianu ${ }^{79}$; University of Florida Brandon Walts ${ }^{80}$, Srikar Chamala ${ }^{80}$, W. Brad Barbazuk ${ }^{80}$; University of Georgia Jingping Li $i^{81}$, Hui Guo ${ }^{81}$, Tae-Ho Lee ${ }^{81}$, Yupeng Wang ${ }^{81}$, Dong Zhang ${ }^{81}$, Andrew H. Paterson (Principal Investigator) ${ }^{81}$, Xiyin Wang (Principal Investigator) ${ }^{81,82}$, Haibao Tang ${ }^{81,83}$; University of Naples "Federico II" Amalia Barone ${ }^{84}$, Maria Luisa Chiusano ${ }^{84}$, Maria Raffaella Ercolano ${ }^{84}$, Nunzio D'Agostino ${ }^{84}$, Miriam Di Filippo ${ }^{84}$, Alessandra Traini ${ }^{44}$, Walter Sanseverino ${ }^{84}$, Luigi Frusciante (Principal Investigator) ${ }^{84}$; University of Nottingham Graham B. Seymour (Principal Investigator) ${ }^{85}$; University of Oklahoma Mounir Elharam ${ }^{86}$, Ying Fu ${ }^{86}$, Axin Hua ${ }^{86}$, Steven Kenton ${ }^{86}$, Jennifer Lewis ${ }^{86}$, Shaoping Lin ${ }^{86}$, Fares Najar ${ }^{86}$, Hongshing Lai ${ }^{86}$ Baifang Qin ${ }^{86}$, Chunmei Qu ${ }^{86}$, Ruihua Shi ${ }^{86}$, Douglas White ${ }^{86}$, James White ${ }^{86}$, Yanbo Xing $^{86}$, Keqin Yang ${ }^{86}$, Jing Yi ${ }^{86}$, Ziyun $\mathrm{Yao}^{86}$, Liping Zhou ${ }^{86}$, Bruce A. Roe (Principal Investigator) ${ }^{86}$; University of Padua Alessandro Vezzi ${ }^{87}$, Michela D'Angelo ${ }^{87}$, Rosanna Zimbello ${ }^{87}$, Riccardo Schiavo ${ }^{87}$, Elisa Caniato ${ }^{87}$, Chiara Rigobello $0^{87}$ Davide Campagna ${ }^{87}$, Nicola Vitulo ${ }^{87}$, Giorgio Valle (Principal Investigator) ${ }^{87}$; University of Tennessee Health Science Center David R. Nelson ${ }^{88}$; University of Udine Emanuele De Paoli ${ }^{89}$; Wageningen University Dora Szinay ${ }^{90,91}$, Hans H. de Jong (Principal Investigator) ${ }^{90}$, Yuling Bai ${ }^{91}$, Richard G. F. Visser ${ }^{91}$, René M. Klein Lankhorst (Principal Investigator) ${ }^{92}$; Wellcome Trust Sanger Institute Helen
Beasley ${ }^{93}$, Karen McLaren ${ }^{93}$, Christine Nicholson ${ }^{93}$, Claire Riddle ${ }^{93}$; Ylichron SrL

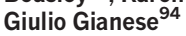

${ }^{1}$ Kazusa DNA Research Institute, 2-6-7 Kazusa-kamatari, Kisarazu, Chiba 292-0818, Japan. ${ }^{2} 454$ Life Sciences, a Roche company, 15 Commercial Street, Branford, Connecticut 06405, USA. ${ }^{3}$ Amplicon Express Inc., 2345 Hopkins Court, Pullman, Washington 99163, USA. ${ }^{4}$ Beijing Vegetable Research Center, Beijing Academy of Agriculture and Forestry Sciences, Beijing 100097, China. ${ }^{5}$ National Center for Gene Research, Chinese Academy of Sciences, Shanghai 200233, China. ${ }^{6}$ BGI-Shenzhen, Shenzhen 518083, China. "BMR-Genomics SrL, via Redipuglia 21/A, 35131 Padova, Italy. ${ }^{8}$ Boyce Thompson Institute for Plant Research, Tower Road, Cornell University campus, Ithaca, New York 14853, USA. ${ }^{9}$ Centre for BioSystems Genomics, PO Box 98, 6700 AB Wageningen, The Netherlands. ${ }^{10}$ Centro Nacional de Análisis Genómico (CNAG), C/ Baldiri Reixac 4, Torre I, 08028 Barcelona, Spain. ${ }^{11}$ Department of Vegetable Science, College of Agronomy and Biotechnology, China Agricultural University, No. 2 Yuanmingyuan Xi Lu, Haidian District, Beijing 100193, China. ${ }^{12}$ Key Laboratory of Horticultural Crops Genetic Improvement of Ministry of Agriculture, Sino-Dutch Joint Lab of Horticultural Genomics Technology, Institute of Vegetables and Flowers, Chinese Academy of Agricultural Sciences, Beijing 100081, China. ${ }^{13}$ State Key Laboratory of Plant Genomics and National Centre for Plant Gene Research, Institute of Genetics and Developmental Biology, Chinese Academy of Sciences, Beijing 100101, China. ${ }^{14}$ Wuhan Botanical Garden, Chinese Academy of Sciences, Wuhan 430074, China. ${ }^{15}$ State Key Laboratory of Plant Cell and Chromosome Engineering and National Center for Plant Gene Research, Institute of Genetics and Developmental Biology, Chinese Academy of Sciences, Beijing 100101, China. ${ }^{16}$ Laboratory of Molecular and Developmental Biology and National Center for Plant Gene Research, Institute of Genetics and Developmental Biology, Chinese Academy of Sciences, Beijing 100080, China. ${ }^{17}$ Cold Spring Harbor Laboratory, One Bungtown Road, Cold Spring Harbor, New York 11724, USA.

${ }^{18}$ Department of Biology, Colorado State University, Fort Collins, Colorado 80523, USA. ${ }^{19}$ Department of Agronomy, National Taiwan University, Taipei 107, Taiwan.

${ }^{20}$ Department of Plant Biology, Cornell University, Ithaca, New York 14853, USA

${ }^{21}$ Genome Bioinformatics Laboratory. Center for Genomic Regulation (CRG), University Pompeu Fabra, Barcelona 08003, Spain. ${ }^{22}$ Department of Plant Systems Biology, VIB. Department of Plant Biotechnology and Bioinformatics, Ghent University, Technologiepark 927, 9052 Gent, Belgium. ${ }^{23}$ Faculty of Agriculture, The Hebrew University of Jerusalem, PO Box 12, Rehovot 76100, Israel. ${ }^{24}$ Institute of Industrial Crops, Heilongjiang Academy of Agricultural Sciences, Harbin 150086, China. ${ }^{25}$ Institute for Bioinformatics and Systems Biology (MIPS), Helmholtz Center for Health and Environment, Ingolstädter Landstr. 1, D-85764 Neuherberg, Germany. ${ }^{26}$ College of Horticulture, Henan Agricultural University, Zhengzhou 450002, China. ${ }^{27}$ National Key Laboratory of Crop Genetic Improvement, Huazhong Agricultural University, Wuhan 430070, China. ${ }^{28}$ Department of Life Sciences, Imperial College London, London SW7 $1 \mathrm{AZ}$, UK. ${ }^{29} \mathrm{NRC}$ on Plant Biotechnology, Indian Agricultural Research Institute, New Delh 110 012, India. ${ }^{30}$ INRA, UR1052 Génétique et amélioration des fruits et légumes, BP 94 84143 Monfavet Cedex, France. ${ }^{31}$ INRA, Biologie du Fruit et Pathologie, 71 rue E. Bourleaux, 33883 Villenave d'Ornon, France. ${ }^{32}$ Unité de Biométrie et d'Intelligence Artificielle UR 875, INRA, F-31320 Castanet-Tolosan, France. ${ }^{33}$ INRA-CNRGV BP52627, 31326 Castanet-Tolosan, France. ${ }^{34}$ Plateforme bioinformatique Genotoul, UR875 Biométrie et Intelligence Artificielle, INRA, 31326 Castanet-Tolosan, France. ${ }^{35}$ Institut National Polytechnique de Toulouse - ENSAT, Université de Toulouse, Avenue de l'Agrobiopole BP 32607, 31326 Castanet-Tolosan, France. ${ }^{36}$ Instituto de Biología Molecular y Celular de Plantas (CSIC-UPV), Ciudad Politecnica de la Innovación, escalera 8E, Ingeniero Fausto Elios s/n, 46022 Valencia, Spain. ${ }^{37}$ Instituto de Hortofruticultura Subtropical y Mediterránea "La Mayora", Universidad de Malaga - Consejo Superior de Investigaciones Cientificas (IHSM-UMA-CSIC), 29750 Algarrobo-Costa (Málaga), Spain ${ }^{38}$ Instituto de Biotecnología, PO Box 25, B1712WAA Castelar, Argentina. ${ }^{39}$ Institute for Biomedical Technologies, National Research Council of Italy, Via F. Cervi 93, 20090 Segrate (Milano), Italy. ${ }^{40}$ Institute of Plant Genetics, Research Division Portici, National Research Council of Italy, Via Università 133, 80055 Portici (Naples), Italy. ${ }^{41}$ ENEA Casaccia Research Center, Via Anguillarese 301, 00123 Roma, Italy. ${ }^{42}$ Scuola Superiore Sant'Anna, Piazza Martiri della Libertà 33 - 56127 Pisa, Italy. ${ }^{43}$ ENEA, Trisaia Research Center, S.S. Ionica - Km 419.5, 75026 Rotondella (Matera), Italy. ${ }^{44}$ James Hutton Institute, Invergowrie, Dundee DD2 5DA, UK. ${ }^{45}$ Barcelona Supercomputing Center, Nexus II Building, c/ Jordi Girona, 29, 08034 Barcelona, Spain. ${ }^{46}$ Institute of Research in Biomedicine, c/ Josep Samiter 1-5, 08028 Barcelona, Spain ${ }^{47}$ ICREA, Pg Lluís Companys, 23, 08010 Barcelona, Spain. ${ }^{48}$ Keygene N.V., Agro Business Park 90, 6708 PW Wageningen, The Netherlands. ${ }^{49}$ Plant Systems Engineering Research Center, Korea Research Institute of Bioscience and Biotechnology, Daejeon, 305-806, Republic of Korea. ${ }^{50}$ Life Technologies, 500 Cummings Center, Beverly, Massachusetts 01915, USA ${ }^{51}$ Life Technologies, 25 avenue de la Baltique, BP 96, 91943 Courtaboeuf Cedex 3 France. ${ }^{52}$ Max Planck Institute for Plant Breeding Research, Carl von Linné Weg 10 50829 Cologne, Germany. ${ }^{53}$ School of Agriculture, Meiji University, 1-1-1 Higashi-Mita, Tama-ku, Kawasaki-shi, Kanagawa 214-8571, Japan. ${ }^{54}$ Department of Plant Science and Plant Pathology, Montana State University, Bozeman, Montana 59717, USA. ${ }^{55}$ NARO Institute of Vegetable and Tea Science, 360 Kusawa, Ano, Tsu, Mie 514-2392, Japan. ${ }^{56}$ National Institute of Plant Genome Research, New Delhi 110067 , India. ${ }^{57}$ Plant Research International, Business Unit Bioscience, Droevendaalsesteeg 1, 6708 PB Wageningen, The Netherlands. ${ }^{58}$ Institute of Plant Genetic Engineering, Qingdao Agricultural University, Qingdao 266109, China. ${ }^{59}$ Roche Applied Science, D-82377 Penzberg, Germany. ${ }^{6}$ Seoul National University, Department of Plant Science and Plant Genomics and Breeding Institute, Seoul 151-921, Republic of Korea. ${ }^{61}$ Seoul National University, Department of Agricultural Biotechnology, Seoul 151-921, Republic of Korea ${ }^{62}$ Seoul National University, Crop Functional Genomics Center, College of Agriculture and Life Sciences, Seoul 151-921, Republic of Korea. ${ }^{63} \mathrm{High}$-Tech Research Center, Shandong Academy of Agricultural Sciences, Jinan, 250000 Shandong, China. ${ }^{64}$ Institute of Vegetables, Shandong Academy of Agricultural Sciences, Jinan, 250100 Shandong, 
China. ${ }^{65}$ School of Life Sciences, Sichuan University, Chengdu, Sichuan 610064 , China. ${ }^{66}$ Sistemas Genomicos, Parque Tecnológico de Valencia, Ronda G. Marconi, 6, 46980 Paterna (Valencia), Spain. ${ }^{67}$ College of Horticulture, South China Agricultural University, 510642 Guangzhou, China. ${ }^{68}$ Syngenta Biotechnology, Inc. 3054 East Cornwallis Road, Research Triangle Park, North Carolina 27709 Durham, USA. ${ }^{69}$ Norwich Research Park, Norwich NR4 7UH, UK. ${ }^{70}$ Department of Botany, The Natural History Museum, Cromwell Road, London SW7 5BD, UK. ${ }^{71}$ United States Department of Agriculture - Agricultural Research Service, RobertW. Holley Center, Tower Road, Cornell University campus, Ithaca, New York 14853, USA. ${ }^{72}$ Instituto de Hortofruticultura Subtropical y Mediterranea. Departamento de Biologia Molecular y Bioquimica, 29071 Málaga, Spain. ${ }^{73}$ Centre de Regulacio Genomica, Universitat Pompeu Fabra, Dr Aiguader, 88, E-08003 Barcelona, Spain. ${ }^{74}$ Arizona Genomics Institute, BIO-5 Institute for Collaborative Research, School of Plant Sciences, Thomas W. Keating Building, 1657 E. Helen Street, Tucson, Arizona

85721 , USA. ${ }^{75}$ Crop Bioinformatics, Institute of Crop Science and Resource Conservation, University of Bonn, 53115 Bonn, Germany. ${ }^{76}$ Department of Plant and Soil Sciences, and Delaware Biotechnology Institute, University of Delaware, Newark, Delaware 19711, USA.

${ }^{77}$ Interdisciplinary Centre for Plant Genomics and Department of Plant Molecular Biology, University of Delhi South Campus, New Delhi 110 021, India. ${ }^{78}$ University of East Anglia, BIO, Norwich NR4 7TJ, UK. ${ }^{79}$ University of East Anglia, CMP, Norwich NR4 7TJ, UK.

${ }^{80}$ Department of Biology and the UF Genetics Institute, Cancer and Genetics Research Complex 2033 Mowry Road, PO Box 103610, Gainesville, Florida 32610, USA. ${ }^{81}$ Plant
Genome Mapping Laboratory, 111 Riverbend Road, University of Georgia, Athens, Georgia 30602, USA. ${ }^{82}$ Center for Genomics and Computational Biology, School of Life Sciences, and School of Sciences, Hebei United University, Tangshan, Hebei 063000,

China. ${ }^{83} \mathrm{~J}$. Craig Venter Institute, 9704 Medical Center Drive, Rockville, Maryland 20850 USA. ${ }^{84}$ University of Naples "Federico II" Department of Soil, Plant, Environmental and Animal Production Sciences, Via Universita', 100, 80055 Portici (Naples), Italy. ${ }^{55}$ Division of Plant and Crop Sciences, University of Nottingham, Sutton Bonington, Loughborough LE12 5RD, UK. ${ }^{86}$ Department of Chemistry and Biochemistry, Stephenson Research and Technology Center, University of Oklahoma, Norman, Oklahoma 73019, USA. ${ }^{87} \mathrm{CRIBI}$, University of Padua, via Ugo Bassi 58/B, 35131 Padova, Italy. ${ }^{88}$ Department of Microbiology, Immunology and Biochemistry, University of Tennessee Health Science Center, Memphis, Tennessee 38163, USA. ${ }^{89}$ Department of Agriculture and Environmental Sciences, University of Udine, via delle Scienze 208, 33100, Udine, Italy. ${ }^{90}$ Wageningen University, Laboratory of Genetics, Droevendaalsesteeg 1, 6708 PB Wageningen, The Netherlands. ${ }^{91}$ Wageningen University, Laboratory of Plant Breeding, Droevendaalsesteeg 1, 6708 PB Wageningen, The Netherlands. ${ }^{92}$ Wageningen University, Droevendaalsesteeg 1, 6708 PB Wageningen, The Netherlands. ${ }^{93}$ Wellcome Trust Sanger Institute Hinxton, Cambridge CB10 1SA, UK. ${ }^{94}$ Ylichron SrL, Casaccia Research Center, Via Anguillarese 301, 00123 Roma, Italy.

$†$ Present address: Plant Engineering Research Institute, Sejong University, Seoul, 143-747, Republic of Korea. 